\title{
The feasibility of using compression bioimpedance measurements to quantify peripheral edema
}

\author{
Leo Koziol ${ }^{1,2,7}$, John J. Pitre, Jr. ${ }^{3}$, Joseph L. Bull ${ }^{3}$, Robert E. Dodde ${ }^{4}$, Grant Kruger ${ }^{1,5,6}$, Alan Vollmer ${ }^{1,2}$, and
} William F. Weitzel ${ }^{1,2}$

1. Department of Veterans Affairs Medical Center, Ann Arbor, MI, USA

2. Department of Internal Medicine, University of Michigan, Ann Arbor, MI, USA

3. Department of Biomedical Engineering, University of Michigan, Ann Arbor, MI, USA

4. Stryker Corporation, Kalamazoo, MI, USA

5. Department of Mechanical Engineering, University of Michigan, Ann Arbor, MI, USA

6. Department of Anesthesiology, University of Michigan, Ann Arbor, MI, USA

7. E-mail any correspondence to:leok@umich.edu

\begin{abstract}
The accurate assessment of body fluid volume is important in many clinical situations, especially in the determination of "dry weight" in a dialysis setting. Currently, no clinically applicable diagnostic system exists to determine the mechanical properties that accurately characterize peripheral edema in an objective and quantitative manner. We have developed a method for quantifying the impact of compression on the electrical properties of tissue by measuring stress-induced changes in bioimpedance (BIS). Using this method, we simultaneously measured the impedance and mechanical response of a tissue mimicking material (tofu) under both quasi-static and dynamic loading conditions. Our results demonstrate a temporal quantification of viscoelastic properties using a viscoelastic phantom tissue model.
\end{abstract}

Keywords: Bioimpedance, peripheral edema, dry weight, tissue compression, tofu.

\section{Introduction}

Management of patients with fluid overload requires assessment of volume status using patient physical examination findings from the cardiopulmonary exam and examination of the extremities for peripheral edema. Recently, interest has grown in attempts at quantitative assessment of tissue hydration using BIS in many disease states $[1,2,3]$. In addition, the tissue response to stress in the setting of edema has recently been the subject of study as a quantitative tool for edema assessment $[4,5,6]$. The examination for early stage/pitting peripheral edema has been performed for centuries by grasping and pressing on the patient's extremity. Common clinical practice demonstrates the desire for quantitative assessment by describing edema "semi-quantitatively," by grading edema from zero to $4+$ based on the observed indentation of the skin with the examiners fingers [7, 8]. Interestingly, measurements to quantify edema have lagged behind our quantitative assessment routinely performed to assess patient blood pressure, laboratory parameters to quantitatively assess pre-renal states, or newer measures that may be predictive of cardiovascular disease.
Quantifying the extra-cellular component of peripheral edema may be useful in a variety of disease states including end stage renal disease (ESRD) and congestive heart failure (CHF). In ESRD, optimal fluid status management is an integral part of the clinical assessment and determining the patient's dry weight is an important component of the patient's treatment [9]. The current standard method of dry weight determination is clinical judgment, which is often reactive (i.e. setting a weight below which the patient develops hypotension or cramping) and subjective instead of goal-directed and quantitative to achieve defined targets based on validated measures. Several other quantitative methods for dry weight determination have been proposed including the use of biochemical parameters, monitoring changes in inferior vena cava diameter, and the use of BIS measurements [10]. In addition, in the CHF setting, monitored approaches to disease management have shown positive outcomes [11]. In both cardiovascular and renal diseases, the clinical care and financial burdens make improvements in assessment and management of fluid status essential $[12,13]$. We therefore are investigating a quantitative approach to assess edema using a modification of conventional segmental BIS methods $[14,15,16]$.

BIS measurements have been used in various ways to improve the assessment of dry weight in dialysis [10, 17, 18]. Fig. 1 shows graphically the principle behind how the flattening of BIS over time can be used to estimate the arrival at a near-dry weight status in dialysis patients. Dryweight in dialysis has been conventionally defined as the lowest tolerated post dialysis weight achieved via gradual change at which there are minimal signs or symptoms of hypervolemia or hypotension. The use of conventional segmental BIS may be helpful in that it relies on identifying the rate of change in edema with fluid removal that occurs when vascular refilling of fluid decreases during dialysis. This may potentially lead to fewer complications from hypotension and other adverse events. Additional confounding effects limit the adoption of conventional BIS measurements such as contact impedance, fluctuations in sodium concentration, differences in dietary 
salt and water intake, body composition, and sodium sequestering [19, 20, 21, 22]. Shifts in ionic content within tissue can lead to misleading interpretations on the edematous nature of tissue as measurement readings are influenced by both fluid volume and ionic content. In particular, ESRD and CHF patients are prone to varying degrees of hyponatremia that make comparisons of BIS measurements less reliable over time [23].

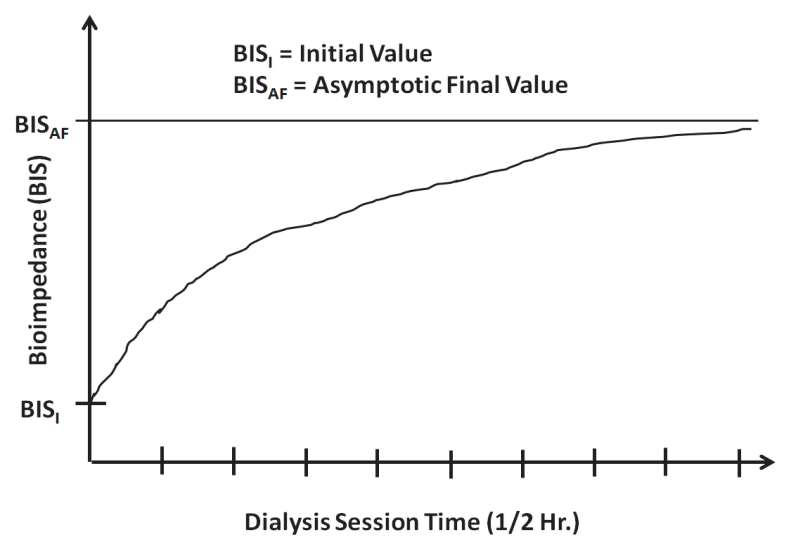

Fig.1: Conventional segmental BIS measurement during a dialysis session, where the measurement is across the lower calf of the patient.

We have overcome these limitations by introducing a provocative stress during BIS measurement. This compression BIS method of monitoring tissue hydration status not only improves accuracy but also incorporates time-dependent viscoelastic properties into the measurement. An accurate assessment of a patient's peripheral edema incorporating a tissue's dynamic response to compression, as would be elicited by physical examination, would provide the clinician with important information.

Compression BIS is a relatively new field, and previous work [24] has sought to link the mechanical and electrical properties of tissue or tissue mimicking materials. Dodde et al. were the first to identify the effect of compression on tissue impedance and fluid levels in porcine spleen $[25,26]$. Their work studied a full range of strains including large strains where cellular collapse was a possibility. Our work concentrated on strains up to approximately $33 \%$, better relating to a clinical situation. Belmont et al. examined the time-dependent electrical properties of a tissue mimicking material (tofu) under a constant strain loading [27]. Their work established the fundamental link between viscoelastic stress relaxation and impedance. The deformation of a viscoelastic material is governed by two phenomena: the deformation of the porous structure and the movement of fluid through that structure. This leads to two characteristic mechanical responses. If a constant strain is impulsively applied, the solid matrix is uniformly compressed and the pore fluid is pressurized. This causes the internal stress in the material to spike. As fluid is driven from the compressed region, the pore pressure "relaxes", and the internal stress decays to a steady-state value. If a constant stress is impulsively applied instead, the porous structure will deform, and the subsequent fluid exudation will result in a gradual increase in the strain. The strain in the material thus "creeps" toward a steady-state value. This fundamental connection between fluid content and tissue mechanics forms the basis for compressive BIS measurements.

Because tissue is composed of both fluid and solid phases, tissue impedance is dependent on a number of factors including the properties of the solid matrix components, internal strain, electrolyte concentrations, and fluid volume. Changes in electrolyte concentration within the tissue are driven by concentration gradients and cantake a long time to reach a steady state. In conventional BIS measurements during a dialysis session, fluid volume changes occur on similar timescales as these concentration changes. This makes it difficult to separate the two effects. Tissue compression generates pressure gradients that modify fluid volume over a much shorter timescale. Changes in fluid volume can therefore be monitored independently of changes in electrolyte concentrations.

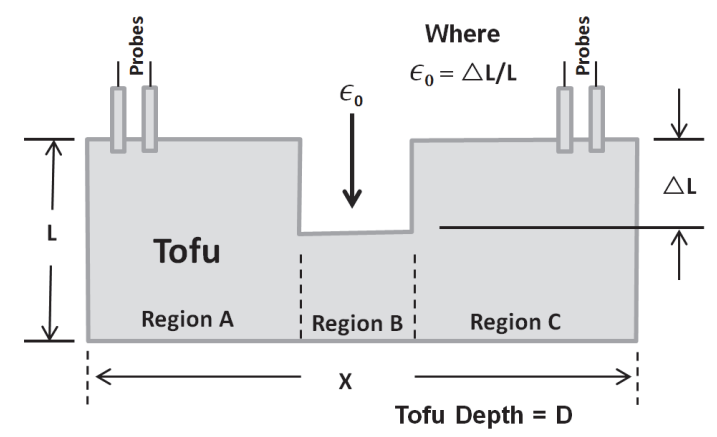

Fig.2: Simple model describing the relationship between resistance and strain in a homogeneous material under compression.

The advantages of incorporating compression into BIS measurements become clear when explained using a simple model. Consider a homogeneous material of dimensions $\mathrm{X}$ $\times \mathrm{L} \times \mathrm{D}$ subjected to a known compressive strain $\epsilon$ (Fig. 2). According to Pouillet's law, the resistance can be defined as $R=\rho(l / A)$, where $\rho$ is the resistivity of the tissue, $A$ is the cross-sectional area, and $l$ is the length of the current path. During compression, the length of the current path remains relatively constant, but the area through which current flows decreases. Following compression, the crosssectional area of the sample can be written as

$$
A=(L-\Delta L) D=L\left(1-\frac{\Delta L}{L}\right) D=L(1-\epsilon) D,
$$

where $D$ is the depth (assumed constant) and $L$ is the height of the material. Substituting this into our previous expression for $R$, we obtain equation 2 where $\epsilon$, the compressive strain is expressed as a ratio of lengths, such as $\mathrm{mm} / \mathrm{mm}$. 


$$
R=\frac{\rho}{D}\left(\frac{1}{1-\epsilon}\right)
$$

Over short time scales (on the order of minutes), the tissue resistivity can be assumed to be a function of fluid volume and strain only, since under compression, we would expect the resistivity to change to that of the solid matrix as fluid exits the region of compression. By measuring both the strain and impedance and applying an appropriate material model, one can recover the fluid volume in the tissue.

In order to better quantify peripheral edema, we have conducted experiments to test the hypothesis that compressive BIS may provide quantitative and reproducible measures of tissue viscoelasticity. This may lead to clinical tools that more accurately determine the extent of edema. In this study, we collected compressive BIS measurements of tofu samples under quasi-static and dynamic loading conditions using a first generation compressive BIS device. We then fit these data with models originally developed to describe the viscoelastic responses of biological materials. Our results demonstrate the feasibility of using compressive BIS as a quantitative tool for estimating fluid volume overload in peripheral edema.

\section{Materials and methods}

Our compressive BIS system (Fig. 3) was based on the tetrapolar BIS circuit designed and developed by Dodde et al. [25, 27, 28, 29]. Our system consisted of a BIS circuit, four platinum leaf probes, an oscilloscope (DSO7032B, Agilent Technologies, Santa Clara, CA), a single-axis programmable mechanical test stand (ESM301L, Mark-10, Copiague, NY), and a computer for automation and data acquisition. Rectangular bars (130 mm long $\times 43 \mathrm{~mm}$ high $\times 20 \mathrm{~mm}$ wide) of extra-firm tofu (Nasoya, Ayer, MA) were soaked in a $0.9 \%$ saline solution for at least 2 days to allow the ion concentration of the tofu phantom to equilibrate near a physiological level.

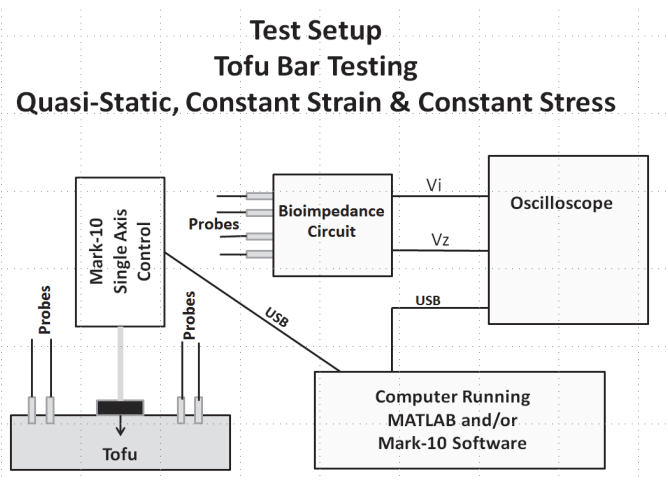

Fig.3: Schematic diagram of the compression BIS system used in this study.

A simple fixture held the tofu bars in place during testing. This fixture featured mesh sides that allowed fluid escaping from the tofu phantom to escape through the sides of the tofu block mimicking the squeezing of fluid out of a compressed region of the body. The single-axis mechanical test stand provided a known compression (either constant strain or constant stress), which was applied to the tofu via a $19 \mathrm{~mm}$ diameter acrylic cylinder. The compression was applied at the center of the tofu bar between two pairs of platinum BIS probes. These probes were positioned $75 \mathrm{~mm}$ apart at opposite ends of the tofu bar. This configuration was meant to closely mimic a possible configuration to be used in future clinical studies. During compression, we measured the impedance of the tofu using a tetrapolar BIS circuit. These data were captured using an oscilloscope and/or a computer, depending on the test. We completed a number of tests to characterize the BIS circuitry and probe design in order to ensure that they could provide accurate and predictable results. Once the circuitry was validated, we conducted compressive BIS experiments on tofu bars under quasi-static and dynamic loading conditions.

\section{Design and Characterization of the BIS Circuit}

In our experiments, we employed a tetrapolar BIS circuit similar to that used by Dodde et al. [25, 28, 30]. This circuit measured tissue impedance by generating a fixed current through a sample of tissue and measuring the voltage drop between two reference points within the tissue. Ohm's law was then used to calculate the impedance. Tetrapolar probes have been previously used in a number of BIS studies and are widely considered to be reliable [27, 31].

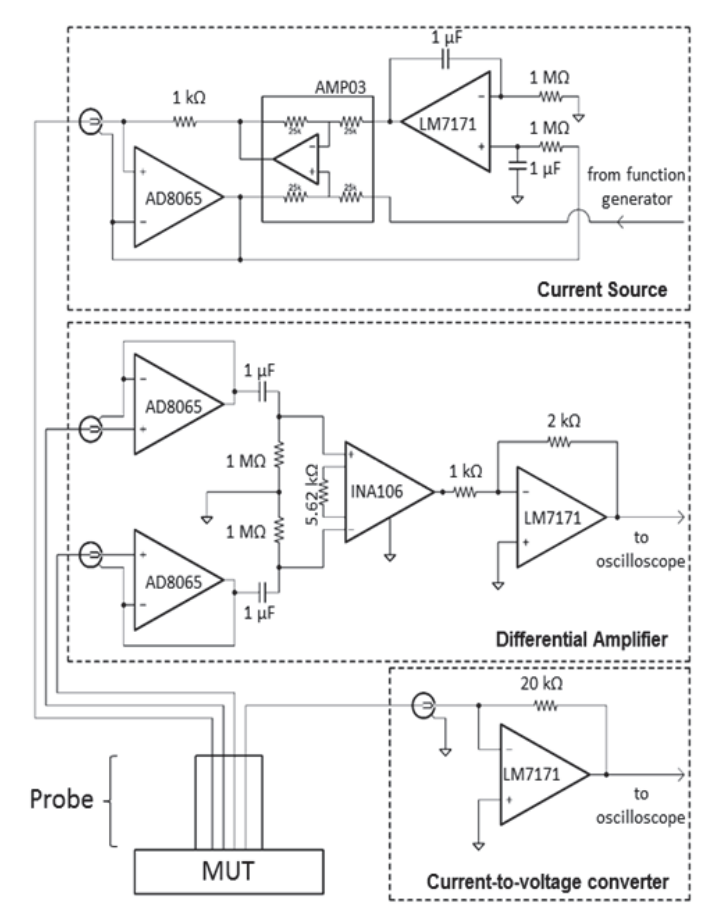

Fig.4: Schematic of the BIS circuitry used in this study.

The schematic for the circuit used in our compression BIS system is shown in Fig. 4. A function generator (AFG3021, Tektronix, Beaverton, OR.) provides a sinusoidal $100 \mathrm{mV}$ reference voltage to control a voltage-controlled current source. This current source injects a $100 \mu \mathrm{A}$ current into the tofu. Voltage readings between two points on the tissue 
are buffered by operational amplifiers connected in voltage follower configurations. The output from the buffer stage (AD8065, Analog Devices, Norwood, MA) is also used to drive the coaxial shield from the BNC extensions of the probes. The buffer stage outputs are differentially amplified using an instrumentation amplifier (INA106, Texas Instruments, Dallas, TX) with a gain of $10 \mathrm{~V} / \mathrm{V}$. The signal is then further amplified using an inverting amplifier with a gain of $2 \mathrm{~V} / \mathrm{V}$ for a total gain of $20 \mathrm{~V} / \mathrm{V}$. The output of this stage is acquired using an oscilloscope. Current output to the tofu is measured via a current-to-voltage converter circuit, which outputs a signal proportional to the current, with a gain of 20,000 V/A.

We verified the BIS circuit functionality by placing a test circuit with known resistance and complex impedance between the BIS leads. The first test circuit consisted of a $1 \%$ tolerance $2000 \Omega$ resistor. The resistor was placed in series with the current source and current-to-voltage converter. We measured the resistance and phase shift for a range of frequencies from $100 \mathrm{~Hz}$ to $100 \mathrm{MHz}$ thus characterizing the usable frequency range of the BIS circuitry.

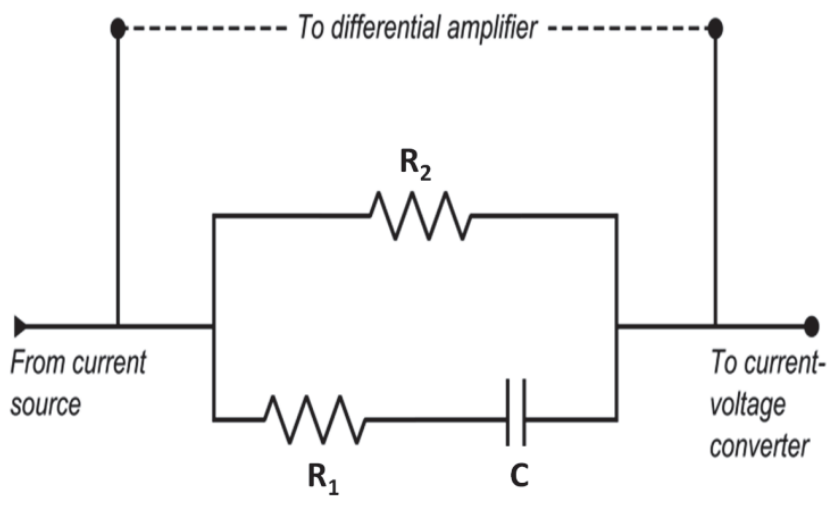

Fig.5: Test circuit used to validate the BIS circuitry and generate a Cole type plot $[32,33,34]$. The circuit consisted of two $1 \%$ $2000 \Omega$ resistors and an $11 \mathrm{nF}$ capacitor $(1 \%$ measured tolerance). The impedance of the test circuit was measured for different current source input frequencies and compared to the theoretical model.

The second test circuit was a linear approximation of a Cole circuit $[32,33,34]$ featuring two $2000 \Omega$ resistors and an 11 $\mathrm{nF}$ capacitor (Fig. 5). We measured the real and imaginary components of the test circuit impedance and compared these values with those predicted by the Cole model. We repeated this test for multiple current source frequencies in the range of $100 \mathrm{~Hz}$ to $2 \mathrm{MHZ}$. For a circuit with only linear elements, the Cole model simplifies to the following resistance-reactance relationship:

$$
\begin{gathered}
\mathcal{R}=\frac{\left(R_{0}-R_{\infty}\right)(\omega \tau)}{1+(\omega \tau)^{2}} \\
X=-R_{\infty}-\frac{R_{0}-R_{\infty}}{1+(\omega \tau)^{2}}
\end{gathered}
$$

where $\mathcal{R}$ is the test circuit resistance, $X$ is the test circuit reactance, $R_{0}(2000 \Omega)$ and $R_{\infty}(1000 \Omega)$ are the zero and infinite frequency limits of the test circuit resistances, respectively, $\omega$ is the frequency of the sinusoidal input, and $\tau=R_{1} C$ is the characteristic time constant on one of the circuit legs in Fig. 5.

\section{Design and Characterization of Bioimpedance Probes}

The studies of Dodde et al. [25] and Belmont et al. [27] used an integrated tetrapolar probe. This probe was composed of a cylindrical composite plastic piece with holes drilled through its length and platinum wires inserted. The end was flat with the measuring probes $3 \mathrm{~mm}$ apart. The entire probe was used to apply compression as well as measure the impedance. Instead, we used two pairs of $3 \mathrm{~mm} \times 3 \mathrm{~mm}$ platinum leaf electrodes (Fig. 6) positioned approximately $75 \mathrm{~mm}$ from each other with compression applied between the two pairs. This design was motivated by two ideas. First, it allowed us to separate the compression and measurement locations and mitigate confounding surface effects caused by fluid exudation near the electrodes. Second, it provided a more practical configuration for integration into our prototype compression BIS system. Platinum was chosen as an electrode material for its low resistivity and to minimize issues with contact resistance and corrosion. The probes were tested by measuring the resistance of saline solutions of varying ion concentrations. A $0.9 \%$ (physiologic) saline solution $(0.1552 \mathrm{~mol} / \mathrm{L}$ solution of $\mathrm{NaCl}$ in water) was diluted with pure water to $100 \%, 50 \%, 33 \%, 25 \%$, and $20 \%$ of the original concentration. The platinum probes were placed in a container of saline solution and held away from the container walls to prevent current crowding effects near boundaries from influencing the measurement. We measured the resistance and phase shift of each saline solution over a range of sinusoidal input frequencies from $100 \mathrm{~Hz}$ to $100 \mathrm{kHz}$.

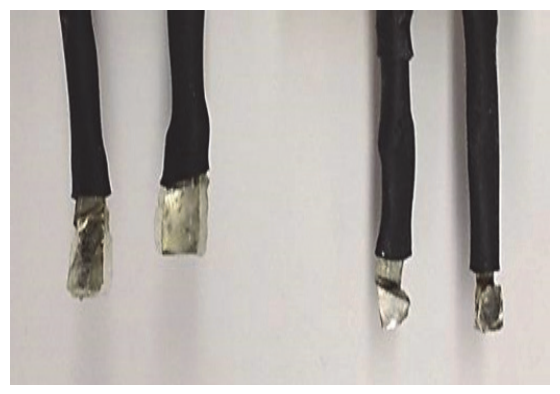

Fig.6: Close-up view of the $3 \mathrm{~mm} \times 3 \mathrm{~mm}$ platinum leaf probes used in this study.

\section{Impedance of Tofu under Quasi-static Loading}

As mentioned previously, viscoelastic materials such as tissue and tofu exhibit a stress relaxation response when subjected to a constant strain. The internal stress within a sample peaks at the onset of compression (induced strain) before relaxing to a steady state. This non-linear process occurs over larger timescales (5 to 10 minutes), but most of 
the transient behavior occurs within the first 2-3 minutes. By waiting for the material to achieve $>90 \%$ steady state conditions, we can obtain impedance measurements under quasi-static conditions. In these experiments, we applied a number of step compressions (constant strains) and allowed the internal stress in the tofu to approach a steady state for each. Tofu bars soaked in $0.9 \%$ saline solution as previously described were placed in a mesh fixture that allowed fluid to flow out of its sides (Fig. 7). Using the BIS circuitry and platinum probes, we first measured the tofu impedance without compression. The tofu was then compressed to a fixed strain, and 5 minutes elapsed before another impedance measurement was taken. Impedance measurements were collected for current source input frequencies of $10 \mathrm{kHz}$ and $20 \mathrm{kHz}$. This compression, waiting increment, and measurement cycle were repeated until the maximum strain (approximately 30\%) was reached. We repeated this experiment for 13 individual tofu bars and averaged the relative strain-induced impedance changes over all of the samples.

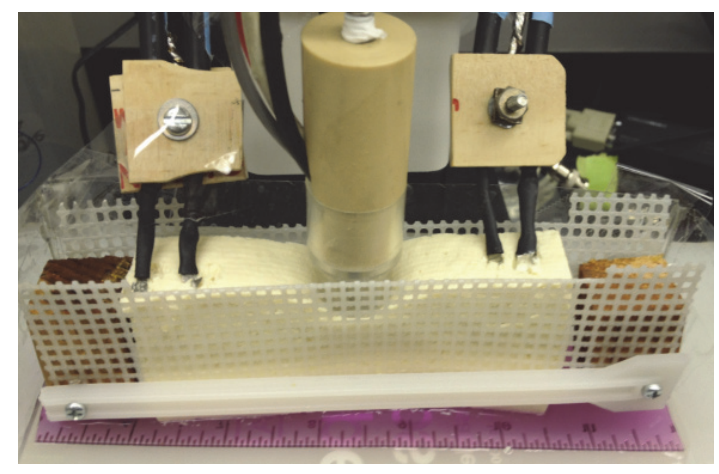

Fig.7: Detailed view of the experimental setup used during impedance measurements for both quasi-static and dynamic loadings.

\section{Impedance of Tofu under Dynamic Loading}

Impedance changes in compressed tissue are caused by the flow of fluid out of the compressed region. There is a strong link therefore between the mechanical and electrical properties of tissue during compression [25]. Biological tissue is typically described as a viscoelastic medium [35, 36]. It follows that compressive BIS measurements should exhibit time-dependent behavior linked to the viscoelastic properties of the tissue in quantifying edema and edema changes during a dialysis session. To test this hypothesis, we performed a series of dynamic compressive BIS tests investigating the relationship between stress-relaxation, creep-compliance, and impedance changes.

Tofu blocks were subjected to a step-like compression and held at a constant strain $(n=13)$ or stress $(n=12)$ for 6 minutes. As before, the tofu blocks were soaked in $0.9 \%$ saline solution to equilibrate to physiologic ion concentrations. In all experiments, the compression rate was limited to a maximum of $100 \mathrm{~mm} / \mathrm{min}$, and the current source input frequency was set to $20 \mathrm{kHz}$. In stress- relaxation experiments, the loading head of the mechanical test stand was lowered to a set position in order to enforce a $33 \%$ strain in the tofu sample. Simultaneous impedance and stress measurements were acquired during and after compression. Impedance readings were digitized using an oscilloscope and streamed to a PC via a MATLAB interface (The Mathworks, Natick, MA). Force measurements from the mechanical test stand were acquired using MesurGauge software (Mark-10, Copiague, NY) and converted to stress using the cross-sectional area of the acrylic cylinder. All experiments were conducted with tofu held at room temperature $\left(+/-0.25{ }^{\mathrm{O}} \mathrm{C}\right.$ tolerance during all the experiments) to minimize any temperature effects. In addition, each tofu sample was subjected to only one loading in order to eliminate permanent mechanical changes from affecting impedance measurements.

Creep-compliance experiments followed a similar protocol to our constant strain compressive BIS experiments. Instead of enforcing a constant strain, the mechanical test stand maintained a $2.5 \mathrm{~N}$ compressive force on the tofu samples. We collected simultaneous BIS and strain measurements for each tofu sample. To measure the strain in the tofu, we first measured the displacement of the test stand loading head using MesurGauge software. These displacement measurements were used to calculate the Green-Lagrange strain, a common strain metric used to describe large deformations (in this case, on the order of $20 \%$ ). As in our stress-relaxation experiments, each tofu sample was subjected to only one loading and the temperature of the tofu was held at room temperature.

\section{Results}

Characterization of the BIS Circuit

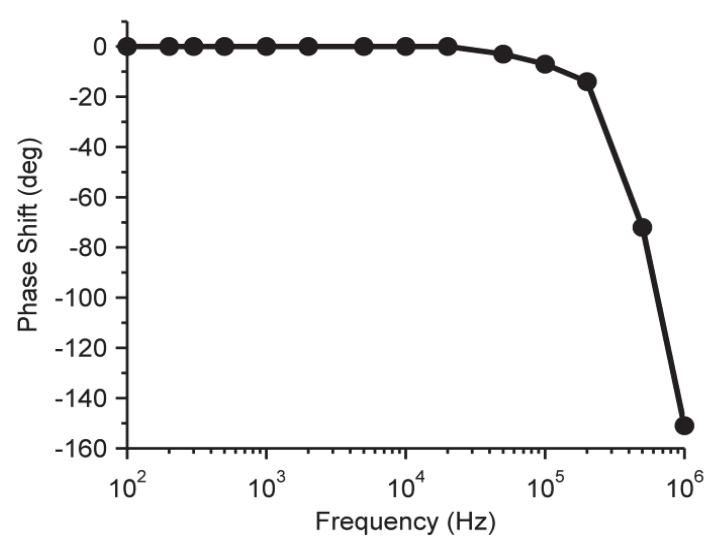

Fig.8: Phase shift measured by the BIS circuitry for a single 2000 resistor test circuit. We observed no phase shift for the frequency range of $500 \mathrm{HZ}$ to $20 \mathrm{KHZ}$. Large phase shifts occurred for frequencies above $100 \mathrm{KHz}$.

For a single $1 \%$ resistor (2000 ohm) test circuit, the BIS circuitry measured a constant resistance with no phase shift for the current source input frequency range of $500 \mathrm{HZ}$ to $20 \mathrm{kHz}$ (Fig. 8). Frequencies in the range $50 \mathrm{kHz}$ to 100 $\mathrm{kHz}$ showed a small phase shift was present. Frequencies upwards of $100 \mathrm{kHz}$ exhibited a much larger phase shift. 
Using the measuring frequencies of $10 \mathrm{KHZ}$ and $20 \mathrm{KHZ}$ allows us to avoid correcting for phase changes in the circuit and keep our calculations simple. In addition, these frequencies keep the effects of polarization [37, 38, 39] between the platinum probes and the tofu over two orders of magnitude below our level of measurement.

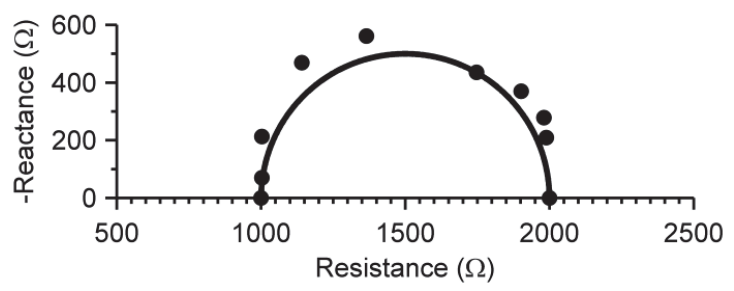

Fig.9: Plot of the measured real and imaginary components of the complex impedance for the test circuit shown in Fig. 5. The experimental data $(\bullet)$ showed reasonable agreement (within 25\%) of the expected theoretical behavior ( - ) (Eq. 3 and 4).

Changing the test circuit to that shown in Fig. 5, and making measurements at frequencies between $500 \mathrm{~Hz}$ and $20.0 \mathrm{KHz}$ yielded data that showed reasonable agreement (within 25\%) with the theoretical model for the real and imaginary resistance as shown in Fig. 9. The data is not conditioned or adjusted.

\section{Characterization of BIS Probes}

Resistance measurements of saline solutions of varying concentrations showed a flat frequency response for the full range of current source input frequencies tested (Fig. 10). Resistance increased as the original $0.9 \%$ saline solution was diluted. No corrosion was apparent on the probe surfaces during or following testing.

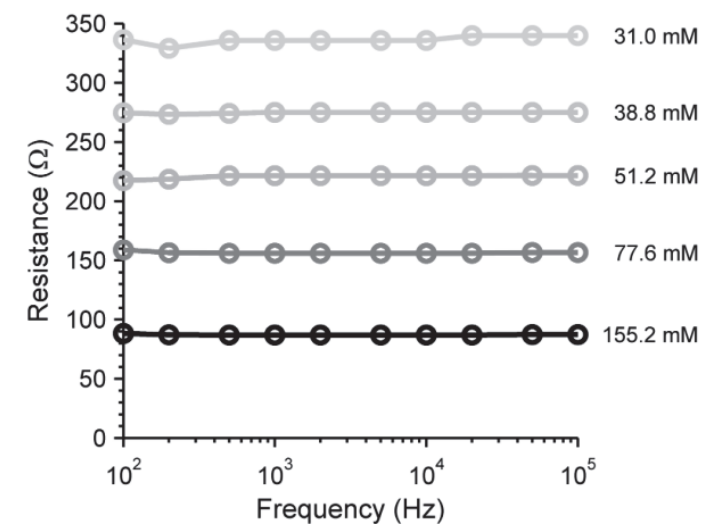

Fig.10: Resistance of $0.9 \%$ saline solution diluted to various fractions of the original concentration $(0.1552 \mathrm{~mol} / \mathrm{L}$ solution of $\mathrm{NaCl}$ in water). Measurements were performed with the BIS circuitry and platinum leaf probes. The electronics exhibit a flat frequency response across the entire spectrum of chosen operating frequencies.

\section{Impedance of Tofu under Quasi-static Loading}

Impedance measurements of tofu under quasi-static loading conditions increased roughly linearly with increasing strain. Since uncompressed impedance differences existed among the individual tofu samples, we computed the percent impedance change (relative to the uncompressed state) for each compression experiment. These percent impedance changes were then averaged across all 13 tofu samples. The mean normalized impedance changes as a function of strain are shown in Figure 11 (for a $10 \mathrm{KHz}$ excitation signal). Larger variations in the normalized impedance measurements were observed as the strain increased. Results at $20 \mathrm{kHz}$ (data not shown) were virtually indistinguishable from the $10 \mathrm{KHz}$ results with a maximum impedance difference of $0.11 \%$. This matched expectations since there is no phase change in the test circuit at these frequencies.

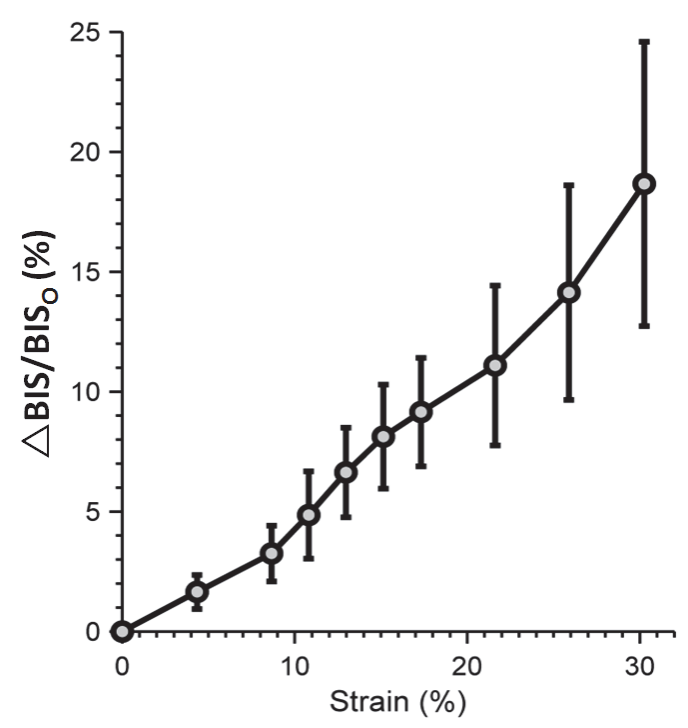

Fig.11: Percent impedance change of tofu blocks under quasistatic loading conditions for a $10 \mathrm{kHz}$ input frequency. As the tofu is compressed, the impedance increases monotonically. Larger variations in the measured impedance are observed at higher strains. Error bars denote \pm one standard deviation from the mean $(n=13)$.

\section{Impedance of Tofu under Dynamic Loading}

Figure 12 shows the stress-relaxation response that is characteristic of viscoelastic materials such as tissue and tofu. The stress rises quickly during the initial compression $(\approx 0-10$ seconds) until the strain limit is reached. The stress then gradually reduces to a steady state as the tofu relaxes. Mechanically, this corresponds to a redistribution of fluid through the porous structure of the tofu. Figure 13 shows the ensemble of normalized changes in BIS along with the ensemble mean and standard deviation. The normalized BIS quickly rises during initial compression $(\approx 0-10$ seconds) then changes gradually as the saline solution redistributes through the tofu. The clear separation of these 
two effects can easily be seen in a parametric plot of the data of normalized change in impedance vs. stress (Fig.14).

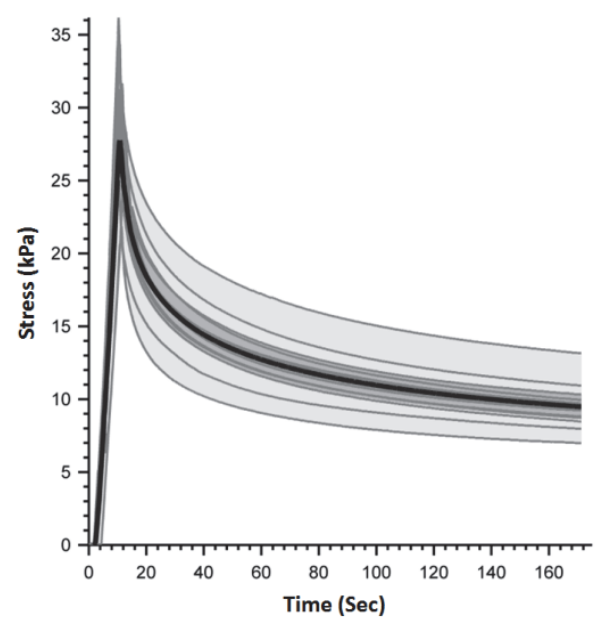

Fig.12: Stress-relaxation response of tofu subjected to a constant strain of $33 \%$. After an initial spike in internal stress, fluid movement within the tofu allows the stress field to approach a steady state. The results of individual experiments (gray lines), the ensemble mean (black line), ensemble envelope (light gray shaded), and \pm one standard deviation from the mean (dark gray shaded) are shown $(\mathrm{n}=13)$.

The impedance increases nearly linearly until the maximum stress is achieved. Then, impedance increases asymptotically as the stress decreases asymptotically to the relaxed state.

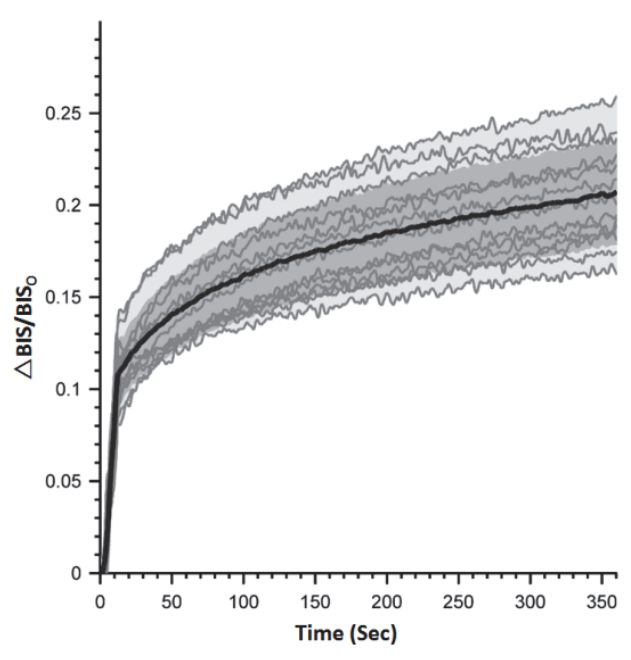

Fig.13: Normalized compression BIS response of tofu subjected to a constant strain of $33 \%$. The impedance rises rapidly during the initial compression then approaches a steady state value. The results of individual experiments (gray lines), the ensemble mean (black line), ensemble envelope (light gray shaded), and \pm one standard deviation from the mean (dark gray shaded) are shown $(\mathrm{n}=13)$.
Under constant stress loading conditions, the tofu samples display a creep response characteristic of viscoelastic materials (Fig. 15). During the initial compression $(\approx 0-10$ seconds), the strain increases sharply. After this, the strain slowly rises asymptotically to a steady state value. A similar trend is observed in the BIS measurements (Fig. 16). A sharp increase in the impedance occurs during the first 10 seconds followed by a much more gradual increase. Note that in contrast to the constant strain results described earlier, the constant stress BIS does not reach a steady state during the 300 second test duration.

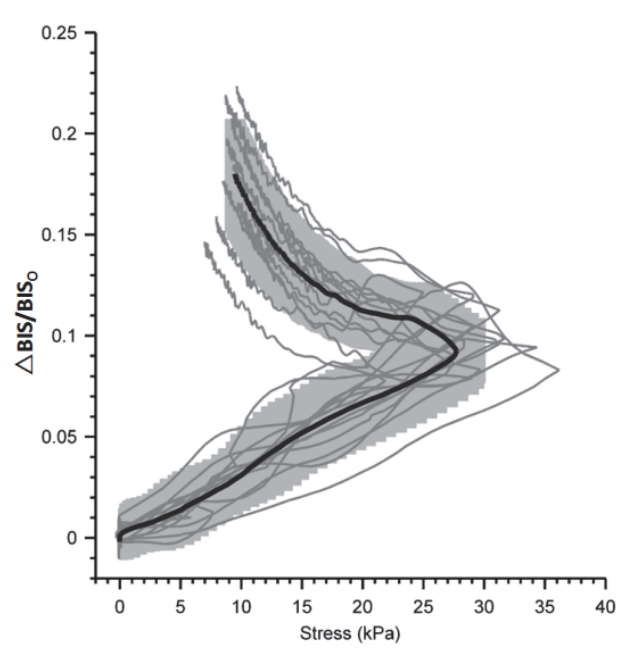

Fig.14: Parametric plot of the stress-relaxation and normalized compression BIS responses of tofu subjected to a constant strain of $33 \%$. The results of individual experiments (gray lines), the ensemble mean (black line), and \pm one standard deviation from the mean (dark gray shaded) are shown $(\mathrm{n}=13)$.

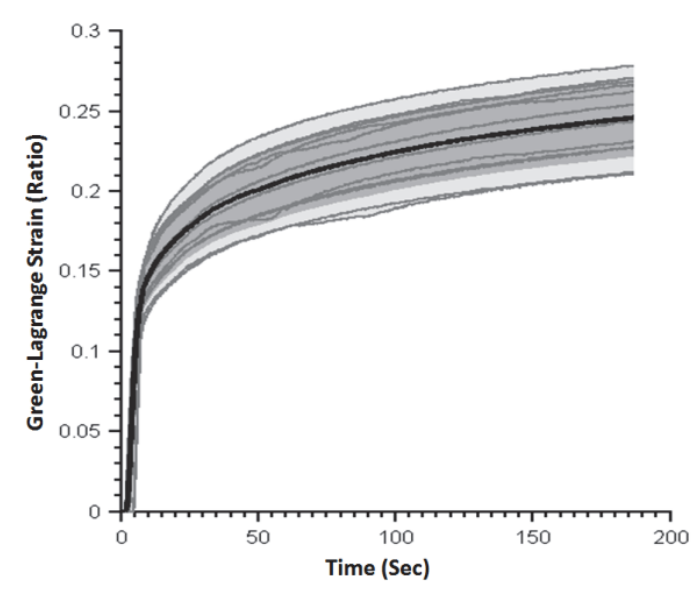

Fig.15: Creep response of tofu subjected to a constant force of $2.5 \mathrm{~N}$ (stress $=8.8 \mathrm{kPa}$ ). After the initial deformation, the strain continues to increase to a steady state. The results of individual experiments (gray lines), the ensemble mean (black line), ensemble envelope (light gray shaded), and \pm one standard deviation. 
The parametric relationship between normalized compressive bioimpedance (BIS) and strain (Fig. 17) shows a slight nonlinearly increasing trend but can still be separated into two distinct regions based on the high frequency noise present as the strain approaches steady state.

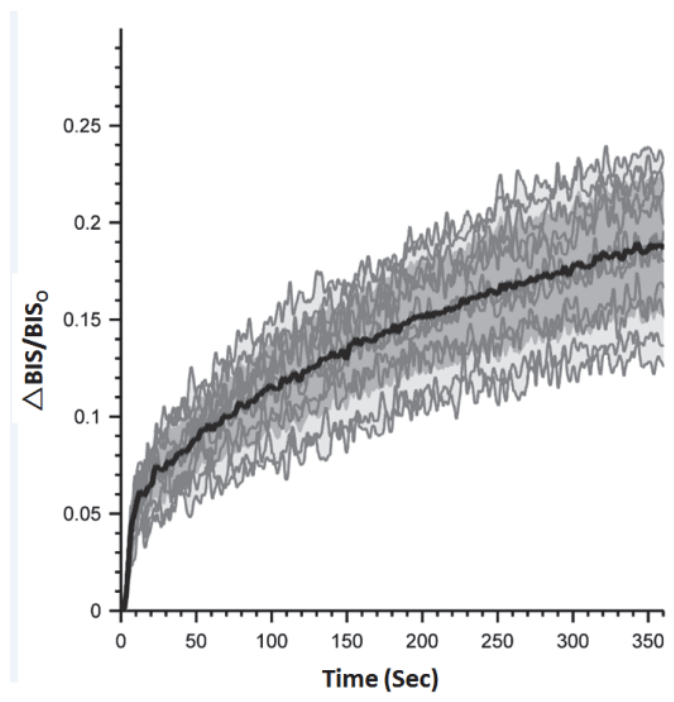

Fig.16: Normalized compression BIS response of tofu subjected to a constant force of $2.5 \mathrm{~N}$ (stress $=8.8 \mathrm{kPa}$ ). After the initial deformation, the impedance rises slowly toward a steady state. The results of individual experiments (gray lines), the ensemble mean (black line), ensemble envelope (light gray shaded), and \pm one standard deviation from the mean (dark gray shaded) are shown $(\mathrm{n}=12)$.

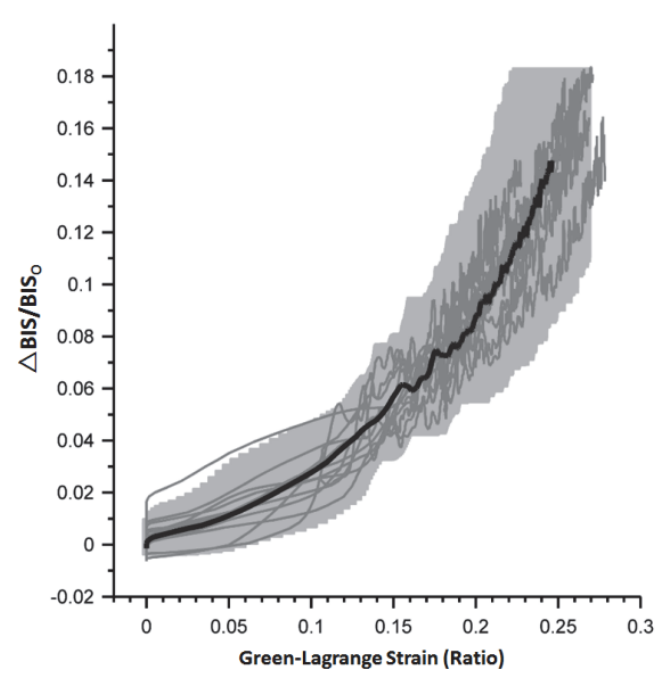

Fig.17: Parametric plot of the creep and normalized compression BIS responses of tofu subjected to a constant force of $2.5 \mathrm{~N}$ (stress $=8.8 \mathrm{kPa}$ ) showing a slight nonlinear increase with increasing strain. The results of individual experiments (gray lines), the ensemble mean (black line), ensemble envelope (light gray shaded), and \pm one standard deviation from the mean (dark gray shaded) are shown $(\mathrm{n}=12)$.

To further characterize the time-dependent changes in BIS, we fit the ensemble average BIS data with a series of exponentials similar to those used to describe viscoelastic mechanics (Eq. 5). Before fitting, a time shift was applied to the BIS data to shift the onset of compression to time $\mathrm{t}=$ 0 . The first coefficient $\overline{\Delta Z}_{c}$ was defined as the impedance change corresponding to the cutoff point of the initial steplike loading. Note that $\overline{\Delta Z}$ refers to the instantaneous normalized change in BIS, and $H(t)$ is the Heaviside step function.

$$
\overline{\Delta Z}(t)=\left[\overline{\Delta Z}_{c}+\overline{\Delta Z}_{1}\left(1-e^{-\frac{t}{\tau_{1}}}\right)+\overline{\Delta Z}_{2}\left(1-e^{-\frac{t}{\tau_{2}}}\right)\right] H(t)
$$

To relate the viscoelastic mechanic changes during compression to the time-dependent impedance response, we repeated the fitting procedure for the ensemble average stress-relaxation and creep-compliance data. This technique is commonly used to characterize the mechanical properties of viscoelastic materials. The stress and strain data were fit with a second order generalized Kelvin-Voigt model [36], a commonly used model to describe viscoelastic materials. Analytical expressions exist for both the stress-relaxation response (Eq. 6) and the creep-compliance response (Eq. 7) of this model. They are, respectively,

$$
\begin{gathered}
\sigma(t)=\epsilon_{0}\left[E_{\infty}+E_{1} e^{-\frac{t}{\tau_{1}}}+E_{2} e^{-\frac{t}{\tau_{2}}}\right] H(t) \\
\epsilon(t)=\sigma_{0}\left[\frac{1}{E_{0}}+\frac{1}{E_{1}}\left(1-e^{-\frac{t}{\tau_{1}}}\right)+\frac{1}{E_{2}}\left(1-e^{-\frac{t}{\tau_{2}}}\right)\right] H(t)
\end{gathered}
$$

where $\epsilon_{0}$ is a constant enforced strain, $\sigma_{0}$ is a constant applied stress, and $\mathrm{H}(\mathrm{t})$ is the Heaviside step function. All other parameters are determined by the curve fitting procedure. The curve fits for the stress-relaxation, creepcompliance, and BIS responses (for both constant stress and constant strain loadings) all show good correlation to the experimental data. coefficient compliance and BIS responses (for both constant stress and constant strain loadings) all show good correlation to the experimental data. We used the coefficient of determination $\left(R^{2}\right)$ as a metric for the goodness of fit. In all cases, the $\mathrm{R}^{2}$ value was greater than $95 \%$. The fit coefficients and characteristic time constants for the stress relaxation response and the corresponding BIS measurements are shown in Tables 1 and 2, respectively. The fit coefficients and time constants for the creep response and its corresponding BIS measurements are shown in Tables 3 and 4, respectively.

Table 1. Stress-relaxation coefficients and time constants for tofu under a constant strain of 33\% as shown in Fig. 12.

\begin{tabular}{lrr}
\hline Parameter & Fit Value & $95 \%$ Confidence Bounds \\
\hline$E_{0}(\mathrm{kPa})$ & 29 & $(28.6,29.3)$ \\
$E_{1}(\mathrm{kPa})$ & 31.23 & $(29.64,32.86)$ \\
$E_{2}(\mathrm{kPa})$ & 30.46 & $(29.74,31.20)$ \\
$\tau_{1}(\mathrm{~s})$ & 3.181 & $(2.866,3.496)$ \\
$\tau_{2}(\mathrm{~s})$ & 45.88 & $(43.36,48.4)$ \\
\hline
\end{tabular}


Table 2. BIS coefficients and time constants for tofu under a constant strain of $33 \%$ as shown in Fig. 13.

\begin{tabular}{lrr}
\hline Parameter & Fit Value & $95 \%$ Confidence Bounds \\
\hline$\overline{\Delta Z}_{c}(\%)$ & 10.88 & N/A \\
$\overline{\Delta Z}_{1}(\%)$ & 3.161 & $(2.823,3.498)$ \\
$\overline{\Delta Z}_{2}(\%)$ & 9.565 & $(9.252,9.878)$ \\
$\tau_{1}(\mathrm{~s})$ & 36.94 & $(33.46,40.42)$ \\
$\tau_{2}(\mathrm{~s})$ & 300.6 & $(257.4,343.7)$ \\
\hline
\end{tabular}

Table 3. Creep-compliance coefficients and time constants for tofu under a constant force of $2.5 \mathrm{~N}($ strain $=8.8 \mathrm{kPa})$ as shown in Fig. 15.

\begin{tabular}{lrr}
\hline Parameter & Fit Value & $95 \%$ Confidence Bounds \\
\hline$E_{0}(\mathrm{kPa})$ & 89.61 & $(88.66,90.56)$ \\
$E_{1}(\mathrm{kPa})$ & 148 & $(145.4,150.6)$ \\
$E_{2}(\mathrm{kPa})$ & 91.7 & $(91.29,92.11)$ \\
$\tau_{1}(\mathrm{~s})$ & 4.517 & $(4.361,4.673)$ \\
$\tau_{2}(\mathrm{~s})$ & 78.72 & $(78.72,80.15)$ \\
\hline
\end{tabular}

Table 4. BIS coefficients and time constants for tofu under a constant force of $2.5 \mathrm{~N}$ (strain $=8.8 \mathrm{kPa}$ ) as shown in Fig. 16

\begin{tabular}{lrr}
\hline Parameter & Fit Value & $95 \%$ Confidence Bounds \\
\hline$\overline{\Delta Z}_{c}(\%)$ & 4.69 & N/A \\
$\overline{\Delta Z}_{1}(\%)$ & 1.731 & $(1.668,1.793)$ \\
$\overline{\Delta Z}_{2}(\%)$ & 16.83 & $(16.68,16.98)$ \\
$\tau_{1}(\mathrm{~s})$ & 9.905 & $(8.942,10.87)$ \\
$\tau_{2}(\mathrm{~s})$ & 263.7 & $(257.6,269.8)$ \\
\hline
\end{tabular}

\section{Discussion}

Based on these results, it appears there is a large change in bioimpedance with modest stress compared with the bioimpedance changes normally seen clinically $[1,2,39$, 40]. Further, this approach may allow investigators to gather more meaningful information in that the data collected is based on a process that replicates the clinical exam, eliciting information on fluid content based on tissue viscoelastic response to stress. There are potential sources of measurement error as with any BIS measurement, such as variation in contact impedance and patient variations. However, this method has the potential advantage to self correct for contact impedance variation by measuring the relative change in BIS from stress rather than relying solely on the baseline bioimpedance signal.

Future work will need to be conducted to test this approach clinically, and to translate this type of measurement into a procedure suitable for clinical study. We are currently developing methods for quantifying peripheral edema that may be robust in the clinical setting and are preparing to conduct a pilot study to measure the compressive BIS for patients undergoing dialysis.
We hypothesize that if an apparatus were used in the dialysis setting, as the dialysis progresses, the BIS would increase through treatment as fluid is removed corresponding to a reduction in edema as shown in the solid plot in Fig. 19. A segmental BIS measuring device has been previously used in the hemodialysis setting [28, 31, 42]. Measurements could be performed using a constant stress in conjunction with single channel, tetrapolar BIS spectroscopy to generate compressive BIS measurements in the clinic. The data collected could be used for the development of a clinically reliable index of residual mobilizable fluid (edema) that may be clinically robust.

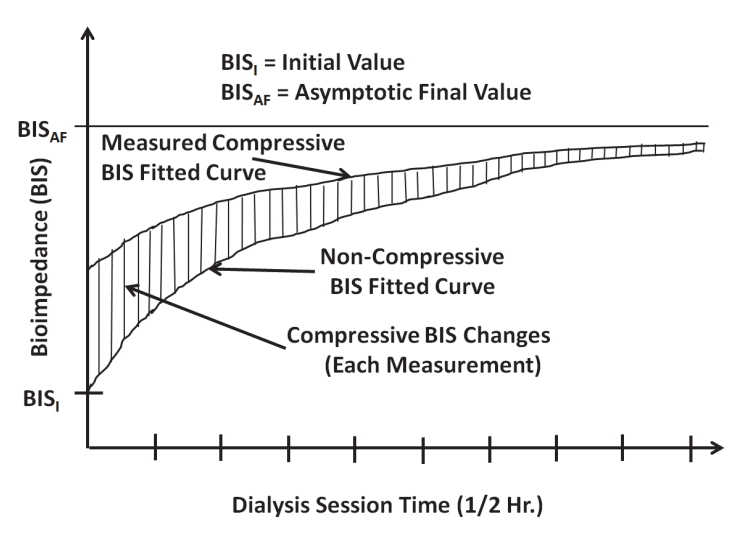

\begin{abstract}
Fig. 18: Anticipated behavior of patient bioimpedance for a single dialysis session. Vertical lines represent compression BIS changes (each measurement). Bottom curve represents aggregate compression BIS during dialysis session. Top curve represents actual BIS as measured during a dialysis session. These data will provide the basis for both dry weight determination (via the asymptotic final value, $\mathrm{BIS}_{\mathrm{AF}}$, and the index of mobilizable fluid (via individual compression time constants).
\end{abstract}

An index or other parameter related to the amount of mobilizable fluid could be determined by fitting a series of curves to the normalized data obtained in the pilot clinical study. In the dialysis setting, these data may help provide the basis for both dry weight determination (via the asymptotic final value, BIS $_{\mathrm{AF}}$, and the index of mobilizable fluid (via individual compression time constants).

Fig. 18 illustrates the anticipated behavior of a patients' BIS obtained during a single dialysis session [39]. Overall, the BIS decays exponentially toward an asymptotic value $\left(\mathrm{BIS}_{\mathrm{AF}}\right)$. For the patient's BIS curves we hypothesize applying a least-squares curve-fitting algorithm to determine the exponential curve that best fits the data as shown in equation 8 .

$$
B I S(t)=B I S_{0}+B I S_{1} e^{-t / \tau}
$$

The exponential curve fit to the envelope BIS data should converge to a single asymptotic final value $\left(\mathrm{BIS}_{\mathrm{AF}}\right)$, which corresponds to the BIS at dry weight. The amount of fluid 
removed by ultrafiltration during the session gives us a conversion factor to relate this asymptotic dry weight BIS to the patient's actual dry weight a hypothesis we plan to test in future clinical studies.

Future laboratory and clinical studies are needed to determine the best approach to improve our understanding of edema and how to best characterize it clinically in order to improve fluid status management and patient outcomes.

\section{Conclusions}

This study has demonstrated a good temporal resolution in the quantification of viscoelastic properties for a tofu phantom model of peripheral edema. This study completes our first set of experiments on device electronics and verification of tetrapolar probes suitable for BIS tissue characterization. Quasi-static BIS vs. strain tests completed on a soft tissue-mimicking phantom material (tofu) yielded repeatable normalized BIS changes with quantified strain. We have characterized the dynamic stress and strain parameters as well as demonstrated repeatable normalized BIS changes of our soft tissue-mimicking phantom material (tofu). Analysis of the data has allowed us to develop normalized parameters with $95 \%$ confidence levels on the curve fitting performed. These experiments and the resulting parameters and time constants have laid the groundwork for developing an approach to quantifying peripheral edema, with future efforts aimed at improving fluid status management and ultimately patient outcomes.

\section{Acknowledgments}

This work was supported by a Merit Review award I01 BX001684 from the United States (U.S.) Department of Veterans Affairs Research and Development Program.

\section{References}

1. Di Somma S, De Berardinis B, Bongiovanni C, Marino R, Ferri E, Alfei B. Use of BNP and Bioimpedance to Drive Therapy in Heart Failure Patients. Congestive Heart Failure. 2010; 16 (Suppl 1): S56-61. http://dx.doi.org/10.1111/j.1751-7133.2010.00162.x

2. McDonald K. Monitoring fluid status at the outpatient level: the need for more precision. Congestive Heart Failure. 2010; 16(4) (Suppl 1): S52-S55. http://dx.doi.org/10.1111/j.1751-7133.2010.00171.x

3. Pirlich M, Schütz T, Ockenga J, Biering H, Gerl H, Schmidt et al. Improved assessment of body cell mass by segmental bioimpedance analysis in malnourished subjects and acromegaly. Clinical Nutrition. 2003; 23(2): 167-174. Erratum in: Clinical Nutrition. 2004; 23(2): 285-286. http://dx.doi.org/10.1054/clnu.2002.0617

4. Czerniec SA, Ward LC, Lee MJ, Refshauge KM, Beith J, Kilbreath SL. Segmental measurement of breast cancerrelated arm lymphoedema using perometry and bioimpedance spectroscopy. Supportive Care in Cancer. 2011; 19(5): 703710. http://dx.doi.org/10.1007/s00520-010-0896-8
5. Stokes IAF, Laible JP, Gardner-Morse MG, Costi JJ, Latridis JC. Refinement of Elastic, Poroelastic, and Osmotic Tissue Properties of Intervertebral Disks to Analyze Behavior in Compression. Annals of Biomedical Engineering. 2011; 39(1): 122-133. http://dx.doi.org/10.1007/s10439-010-0140-1

6. Berry GP, Bamber JC, Mortimer PS, Bush NL, Miller NR, Barbone PE. The spatio-temporal strain response of oedematous and nonoedematous tissue to sustained compression in vivo. Ultrasound in Medicine \& Biology. 2008; 34(4): 617-629. http://dx.doi.org/10.1016/j.ultrasmedbio.2007.10.007

7. O'Sullivan SB, Schmitz TJ. Physical rehabilitation: assessment and treatment. 5th ed. Philadelphia, Pa: FA Davis Company; 2001.

8. Hogan MA, Madayag TM. Medical-Surgical Nursing: Reviews \& Rationales. Upper Saddle River, NJ: Pearson Prentice Hall; 2004.

9. Agarwal R, Andersen MJ, Pratt JH. On the importance of pedal edema in hemodialysis patients. Clinical Journal of the American Society of Nephrology. 2008; 3(1): 153-158. http://dx.doi.org/10.2215/CJN.03650807

10. Zhu F, Kuhlmann MK, Kotanko P, Seibert E, Leonard EF, Levin NW. A method for the estimation of hydration state during hemodialysis using a calf bioimpedance technique. Physiological measurement. 2008; 29(6): S503-16. http://dx.doi.org/10.1088/0967-3334/29/6/S42.

11. Lowe A, Jones RW, Harrison MJ. The graphical presentation of decision support information in an intelligent anesthesia monitor. Artificial Intelligence in Medicine. 2001; 22(2): 173-191. http://dx.doi.org/10.1016/S0933-3657(00)00106-8

12. Imhoff M, Kuhls S, Gather U, Fried R. Smart alarms from medical devices in the OR and ICU. Best Practice \& Research Clinical Anaesthesiology. 2009; 23(1): 39-50. http://dx.doi.org/10.1016/j.bpa.2008.07.008

13. Kotanko P, Levin NW, Zhu F. Current state of bioimpedance technologies in dialysis. Nephrology Dialysis

Transplantation. 2008; 23: 808-812. http://dx.doi.org/10.1093/ndt/gfm889

14. Zhu F, Schneditz D Levin NW. Sum of segmental bioimpedance analysis during ultrafiltration and hemodialysis reduces sensitivity to changes in body position. Kidney International. 1999; 56(2): 692-699. http://dx.doi.org/10.1046/j.1523-1755.1999.00588.x

15. Schoeller DA, Alon A, Manekas D, Mixson LA, Lasseter KC, Noonan GP, et al. Segmental Bioimpedance for Measuring Amlodipine-Induced Pedal Edema: A PlaceboControlled Study. Clinical Therapeutics. 2012; 34(3): 580592. http://dx.doi.org/10.1016/j.clinthera.2012.01.018

16. Cornish BH, Jacobs A, Thomas BJ, Ward LC. Optimizing electrode sites for segmental bioimpedance measurements. Physiol. Meas. 1999; 20(3): 241-250.

17. Chongthanakorn K, Tiranathanagul K, Susantitaphong P, Praditpornsilpa K, Eiam-Ong S. Effective determination of dry weight by intradialytic bioimpedance analysis in hemodialysis. Blood Purification. 2009; 27(3): 235-241. http://dx.doi.org/10.1159/000199428 
18. Lowe A, Jones RW, Harrison MJ. The graphical presentation of decision support information in an intelligent anesthesia monitor. Artificial Intelligence in Medicine. 2001; 22(2): 173-191. http://dx.doi.org/10.1016/S0933-3657(00)00106-8

19. Titze J, Luft FC, Bauer K, Dietsch P, Lang R, Veelken R, et al., Extrarenal $\mathrm{Na}+$ balance, volume, and blood pressure homeostasis in intact and ovariectomized deoxycorticosterone-acetate salt rats. Hypertension. 2006; 47(6): 1101-1107. http://dx.doi.org/10.1161/01.HYP.0000221039.17735.1a

20. Titze J, Shakibaei M, Schafflhuber M, Schulze-Tanzil G, Porst M, Schwind KH, et al. Glycosaminoglycan polymerization may enable osmotically inactive $\mathrm{Na}+$ storage in the skin. American Journal of Physiology-Heart and Circulatory Physiology. 2004; 287(1): H203-208. http://dx.doi.org/10.1152/ajpheart.01237.2003

21. Ebah LM, Wiig H, Dawidowska I, O'Toole C, Summers A, Nikam M, et al. Subcutaneous interstitial pressure and volume characteristics in renal impairment associated with edema. Kidney International. 2013; 84: 980-988. http://dx.doi.org/10.1038/ki.2013.208

22. Rees AE, Ward LC, Cornish BH, Thomas BJ. Sensitivity of multiple frequency bioelectrical impedance. Physiol. Meas. 1999; 20: 349-362. http://dx.doi.org/10.1088/0967-3334/20/4/302

23. Gabrielli D, Kristal B, Katzarski K, Youssef M, Hachache T, Lopot F, et al., Improved intradialytic stability during haemodialysis with blood volume-controlled ultrafiltration. Journal of Nephrology. 2009; 22(2): 232-240.

24. Kohandel M, Sivaloganathan S, and Tenti G. Estimation of the quasi-linear viscoelastic parameters using a genetic algorithm. Mathematical and computer modeling. 2008; 47(3-4): 266-270. http://dx.doi.org/10.1016/j.mcm.2007.04.006

25. Dodde RE, Bull J, and Shih A. Bioimpedance of soft tissue under compression. Physiological Measurement. 2012; 33: 1095. http://dx.doi.org/10.1088/0967-3334/33/6/1095

26. Keshtkar A. The effect of applied pressure on the electrical impedance of the bladder tissue using small and large probes. Journal of Medical Engineering and Technology. 2008; 32(6): 505-511. http://dx.doi.org/10.1080/03091900701507456

27. Belmont B, Dodde RE, Shih AJ. Impedance of tissuemimicking phantom material under compression. Journal of Electrical Bioimpedance. 2013; 4: 2-12. http://dx.doi.org/10.5617/jeb.443

28. Nordbotten BJ. Bioimpedance Measurements Using the Integrated Circuit AD5933. [dissertation] Oslo, Norway: Department of Physics, University of Oslo; 2008.

29. Dodde R., Kruger, G., Shih, A. Design of Bioimpedance Spectroscopy Instrument with Compensation Techniques for Soft Tissue Characterization. ASME Journal of Medical Devices. Forthcoming 2014.
30. Grimnes S, and Martinsen OG. Bioimpedance and bioelectricity basics. $2^{\text {nd }}$ ed. Oxford, UK: Academic Press; 2008.

31. Steendijk P, Baan J, Mur G, Van Der Velde ET. The fourelectrode resistivity technique in anisotropic media: theoretical analysis and application on myocardial tissue in vivo. IEEE Transactions on Biomedical Engineering. 1993; 40(11): 1138-1148. http://dx.doi.org/10.1109/10.245632

32. Cole KS, Cole RH. Dispersion and absorption in dielectrics I. Alternating current characteristics. The Journal of Chemical Physics. 1941; 9: 341-351. http://dx.doi.org/10.1063/1.1750906

33. Elwakil AS, Maundy B. Extracting the Cole-Cole impedance model parameters without direct impedance measurement. Electronics Letters. 2010; 46: 1367-1368. http://dx.doi.org/10.1049/el.2010.1924

34. Cole, K.S. Permeability and impermeability of cell membranes for ions. Cold Spring Harb. Symp. Quant. Biol. 1940; 8: 110-122. http://dx.doi.org/10.1101/SQB.1940.008.01.013

35. Hanai T. Electrical properties of emulsions. In: Sherman DH (ed) Emulsions science. 1994; Academic Press, London, 354477.

36. Fung YC. Biomechanics: Mechanical Properties of Living Tissues, 2nd ed. New York, NY: Springer-Verlag; 1993. http://dx.doi.org/10.1007/978-1-4757-2257-4

37. Onaral B, Schwan HP. Linear and nonlinear properties of platinum electrode polarization. Part 1: frequency dependence at very low frequencies. Med. \& Biol. Eng. \& Comput. 1982; 20: 299-306. http://dx.doi.org/10.1007/BF02442796

38. Onaral B, Schwan HP. Linear and nonlinear properties of platinum electrode polarization. Part 2: Time domain analysis. Med. \& Biol. Eng. \& Comput. 1983; 21: 210-216. http://dx.doi.org/10.1007/BF02441540

39. Schwan HP. Alternating current electrode polarization. Biophysik. 1966; 3: 181-201. http://dx.doi.org/10.1007/BF01191612

40. Zaluska W, Malecka T, Mozul S, Ksiazek A. Whole body versus segmental bioimpedance measurements (BIS) of electrical resistance $(\mathrm{Re})$ and extracellular volume (ECV) for assessment of dry weight in end-stage renal patients treated by hemodialysis. Przegl Lek. 2004; 61(2): 70-3. In Polish.

41. Jaffrin MY, Morel H. Extracellular volume measurements using bioimpedance spectroscopy - Hanai method and wristankle resistance at $50 \mathrm{kHz}$. Med. Biol Comput. 2009; 47(1): 77-84. http://dx.doi.org/10.1007/s11517-008-0394-z

42. Zhu F, Schneditz D, Wang E, Martin K, Morris AT, and Levin NW. Validation of changes in extracellular volume measured during hemodialysis using a segmental bioimpedance technique. ASAIO J. 1998; 44: 541-545. http://dx.doi.org/10.1097/00002480-199809000-00045 